\title{
Rudolf Höfer (dir.), Kirchenfinanzierung in Europa. Modelle und Trends
}

Innsbruck, Tyrolia Verlagsanstalt Gm, Coll. « Theologie im kulturellen Dialog », Universität Graz, 2014, 248 p.

\section{Isabelle Jonveaux}

\section{(2) OpenEdition}

\section{Journals}

Édition électronique

URL : http://journals.openedition.org/assr/26454

DOI : $10.4000 /$ assr. 26454

ISSN : 1777-5825

Éditeur

Éditions de l'EHESS

Édition imprimée

Date de publication : 31 décembre 2014

Pagination : 215

ISBN : 978-2-7132-2467-6

ISSN : 0335-5985

Référence électronique

Isabelle Jonveaux, « Rudolf Höfer (dir.), Kirchenfinanzierung in Europa. Modelle und Trends », Archives de sciences sociales des religions [En ligne], 168 | 2014, mis en ligne le 29 avril 2015, consulté le 22 septembre 2020. URL : http://journals.openedition.org/assr/26454 ; DOI : https://doi.org/10.4000/ assr.26454

Ce document a été généré automatiquement le 22 septembre 2020

() Archives de sciences sociales des religions 


\section{Rudolf Höfer (dir.), Kirchenfinanzierung in Europa. Modelle und Trends}

Innsbruck, Tyrolia Verlagsanstalt Gm, Coll. « Theologie im kulturellen Dialog », Universität Graz, 2014, 248 p.

Isabelle Jonveaux

\section{RÉFÉRENCE}

Rudolf Höfer (dir.), Kirchenfinanzierung in Europa. Modelle und Trends, Innsbruck, Tyrolia Verlagsanstalt Gm, Coll. « Theologie im kulturellen Dialog », Universität Graz, 2014, $248 \mathrm{p}$. 
1 Nous savons que le financement des Églises diffère selon chaque pays en fonction de divers facteurs historiques et sociaux. Mais savons-nous exactement comment ce financement fonctionne dans les pays européens? Vue à travers le prisme français, cette question est essentiellement étrangère du fait de la privatisation du financement des cultes en France- hormis l'insolite situation de l'Alsace-Lorraine - qui apparaît pourtant comme une quasi-exception dans le paysage européen. Cet ouvrage dirigé par Rudolf Höfer, professeur d'histoire de l'Église à la Faculté de théologie de Graz, et issu d'une conférence tenue dans cette même faculté en octobre 2012, a l'immense mérite de faire le point en neuf

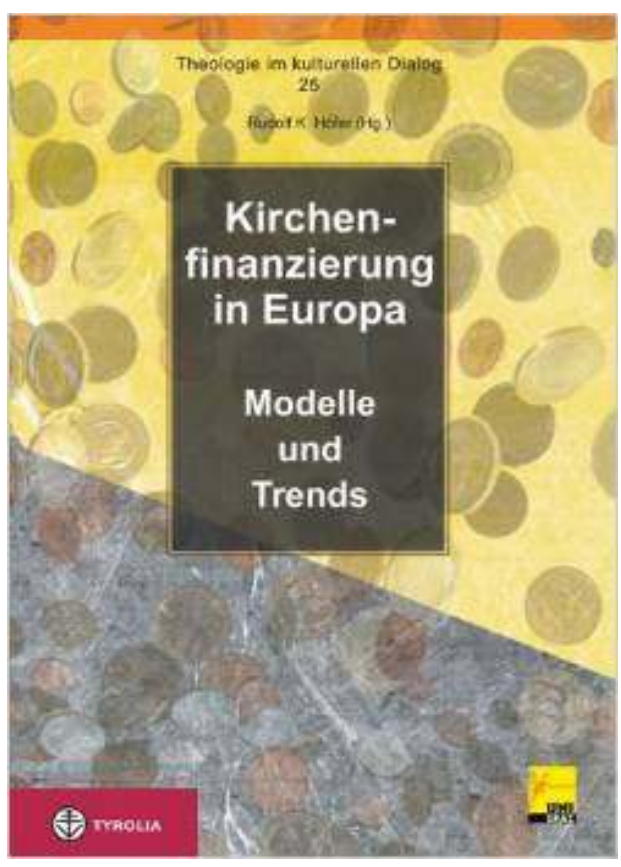
articles sur cette question dans une grande part des pays européens. Est abordé dans ce livre le financement des Églises en Belgique, Allemagne, Italie, Hongrie, Autriche, Royaume-Uni, France, Slovénie et différents pays du Nord que sont l'Islande, le Norvège, la Suède, la Finlande et le Danemark.

2 Si chaque pays possède sa propre organisation concernant le financement des Églisesou des cultes pour utiliser le concept proprement français dont la traduction a posé problème dans le cas de l'article de J.-P. Moisset - trois modèles principaux sont cependant identifiables. Le premier est celui d'un financement de l'Église par le biais officiel de l'État sous la forme d'un impôt. Ce modèle présente deux ramifications: l'impôt de l'Église (Kirchensteuer) auquel est soumis tout contribuable ayant déclaré son appartenance religieuse, en Allemagne ou en Suède par exemple, et l'impôt culturel (Kultursteuer), en Italie par exemple, qui est une part d'impôt que le contribuable peut choisir librement de donner à telle ou telle Église, ou à l'État qui l'utilisera pour une action culturelle ou caritative. Pour l'otto per mille italien, chaque contribuable choisit de donner huit pour mille de son revenu imposable soit à l'une des sept Églises recensées soit à l'État dans un but culturel. Ce principe est relativement similaire en Espagne et en Hongrie. Selon R. Höfer (introduction, p. 8), il est prévu que la Pologne adapte ce système en 2014 et cette évolution est aussi planifiée en Slovaquie et au Liechtenstein. Le second modèle consiste en un financement direct des institutions religieuses par l'État sans que soit explicitement prélevé un impôt spécifique en ce sens. C'est le cas de la Belgique où Jan de Mayer évoque un financement indirect et non pas direct de l'Église (p.9). Enfin, le dernier modèle est celui des contributions volontaires des fidèles, directement versées aux Églises, et qui s'apparente alors à des dons privés, cas que l'on peut trouver en France et en Slovénie, et qui est souvent associé à une séparation de l'Église et de l'État.

3 La question du financement des Églises ne se limite pas à celle des édifices, activités et personnels religieux mais concerne aussi les activités périphériques que sont l'éducation, les actions caritatives et culturelles. Selon les modèles, celles-ci sont 
soumises au même traitement que le cœur religieux de l'ensemble «Église » ou au contraire, comme en France et en Slovénie, peuvent faire l'objet d'un traitement particulier avec participation de l'État alors que les activités cultuelles en soi n'en bénéficient pas. On découvre en effet à la lecture de cet ouvrage un point commun peu connu entre la France et la Slovénie qui est de présenter un modèle quasi identique concernant le financement des cultes, avec globalement un financement privé de la part des fidèles et une participation de l'État concernant les activités périphériques ayant un intérêt social. Lorsque le financement se fait sur la base du volontariat, par les fidèles directement (Denier du Culte français par exemple), ou entre différentes possibilités (Italie), cela entraîne aussi de la part des Églises des campagnes publicitaires en ce sens. En Autriche, un scandale a été déclenché en 2012 en Styrie lorsque l'Église a envoyé un SMS de rappel aux fidèles qui n'avaient pas encore versé leur contribution (Höfer, p.111). Mais il faut aussi souligner, comme le fait Jan de Mayer concernant la Belgique, que contrairement à ce que pense l'opinion publique ou les croyants eux-mêmes, les ordres religieux et monastères sont indépendants de ce système et ne reçoivent, dans aucun des pays cités, une part de ces prélèvements. Dans des pays comme l'Allemagne, ainsi qu'on me l'avait expliqué lors d'une enquête de terrain dans un monastère bénédictin en Bavière, où l'Église catholique est ostensiblement riche - l'affaire de l'évêque de Limbourg a révélé le problème de la richesse de l'Église allemande dans les médias - cette impression peut porter préjudice aux ordres religieux qui reçoivent par là même moins de dons de la part des croyants, persuadés qu'ils sont que leur impôt ecclésial profite aussi à ces communautés. Inversement, avec la suppression de quelque 700 à 800 monastères au XVIII ${ }^{e}$ siècle, l'empereur Joseph II avait constitué un fonds de financement de l'Église dont celle-ci a pu profiter pendant longtemps.

4 La lecture des différentes situations des pays ici représentés montre des déterminants communs qui interviennent dans la question du financement des Églises. Tout d'abord, chaque article témoigne du poids de l'histoire dans l'organisation actuelle, faisant suite aux divers concordats ou nationalisations des biens de l'Église. R. Höfer souligne que le cas autrichien continue finalement de vivre sur la situation instaurée sous le nationalsocialisme. En Slovénie, la dé-nationalisation des biens du clergé depuis l'instauration de l'État indépendant de Slovénie fournit au diocèse de Ljubljana la majeure partie de ses revenus comme contrepartie des préjudices subis lors de la nationalisation (Andrej Saje). En France, la situation actuelle, qu'elle concerne l'Alsace-Lorraine ou le reste de l'hexagone, est aussi issue de plus de deux siècles d'évolution, où le concordat napoléonien ou la séparation de l'Église et de l'État de 1905, ont joué des rôles centraux (J.-P. Moisset).

5 Le financement des Églises pose aussi la question délicate des organisations que l'on désigne sous ce terme et donc de ce que l'État reconnaît comme Église. Cette question devient un enjeu d'autant plus important dans un contexte de pluralité religieuse, susceptible d'évolutions en fonction notamment des mouvements de populations. On arrive alors à une situation dans laquelle le parlement peut reconnaitre la qualité d'« Église » à une communauté religieuse pour qu'elle bénéficie ensuite du financement public. Dans d'autres pays, comme au Royaume-Uni (David Thompson) ou en Suède (Yvonne Maria Werner), on parle d'Église d'État pour la communauté majoritaire et il n'existe pas de réelle séparation entre le pouvoir temporel et le pouvoir spirituel. Dans cette configuration, le monarque est souvent aussi à la tête de l'Église. Dans plusieurs pays du Nord, dont la Suède, c'est aussi le Parlement qui a voté l'accès aux femmes à 
l'ordination dans les Églises luthériennes. La définition des relations entre les/ l'Église(s) et l'État est donc déterminante concernant la question du financement et cette dernière présuppose aussi une définition publique des communautés religieuses.

6 La contribution de Rudolf Höfer mérite qu'on s'y arrête car elle ne se limite pas à décrire le cas autrichien, mais en interroge la pérennité et propose des solutions alternatives. Le système d'impôt prélevé par les Églises (catholiques, évangéliques et vieilles catholiques) selon les déclarations d'appartenance religieuse des citoyens lors de leur inscription dans une ville (Meldezettel) est toujours basé sur des accords datant du national-socialisme. Mais hormis la volonté de rompre avec cet héritage, la question se pose de la pertinence de ce système. Dans la lignée d'autres professeurs de théologie ou évêques qui ont pris position pour cette évolution, Höfer expose les avantages d'un système de contribution culturelle volontaire entre différentes communautés ou causes, expérimenté en Italie. Hormis le fait que sont soumis à ces impôts eux-mêmes les croyants exonérés des autres impôts, ce système de prélèvement pour tout croyant déclaré, entraîne, comme en Allemagne, la délicate question des « sorties de l'Église ", qui n'existe paradoxalement pas dans des pays plus sécularisés ou laïcisés comme la France. L'auteur souligne que l'Autriche est le pays où les « sorties de l'Église » sont les plus élevées en Europe, et il ajoute que la contribution obligatoire à l'Église entraîne une opinion négative vis-à-vis de cette institution. Ce serait selon lui un argument essentiel pour faire évoluer le système vers des contributions volontaires. La question des « sorties de l'Église » - qui s'opèrent sur simple lettre envoyée à l'évêque local - est d'autant plus délicate dans le champ catholique que le baptême est théoriquement indélébile. À cela s'ajoutent aussi les fraudes, qui représentent un pourcentage non négligeable.

7 Sans que ce soit directement l'objet de cet ouvrage, la question du financement permet aussi d'aborder celle de leur affectation entre les différents postes que sont le personnel, la mise en œuvre du culte, le patrimoine immobilier ou enfin les œuvres caritatives. On y apprend ainsi que les prêtres italiens sont soumis pour leur revenu à un système de points (M. Mitterhofer, p. 142), modulé en fonction de l'ancienneté, de la position hiérarchique et de différentes charges. De même, comme le souligne Annamária Schlosser pour le cas de la Hongrie, mais cela concerne aussi différents autres pays, l'Église est exempte d'impôts locaux, ce qui lui voue, d'une certaine manière par son épargne, un revenu supplémentaire. Les graphiques et tableaux de chiffres permettent aussi d'effectuer quelques comparaisons. L'archidiocèse de Ljubljana en Slovénie, par exemple, bénéficie en 2011 de plus de 3,8 millions d'euros de revenus. Pour le diocèse de Vienne, ces revenus s'élèvent à plus de 100 millions en 2010. En Allemagne, chaque diocèse a reçu environ 7,7 millions uniquement des impôts prélevés par l'État. Concernant ces budgets, il faut aussi avoir en tête leur affectation notamment pour le nombre d'employés qu'ils permettent de payer. Le diocèse de Linz en Autriche par exemple ne compte pas moins de 30000 employés.

Si l'on regrette l'absence d'un tableau récapitulatif présentant les différents modèles en fin d'ouvrage et si l'aspect parfois très juridique - mais nécessaire - des contributions rend la lecture plus difficile pour les non spécialistes, il faut souligner encore une fois l'apport réellement riche de cet ouvrage, qui permet un regard général sur des situations très différentes dans l'espace européen. Une traduction serait certainement utile pour rendre plus largement accessible cette étude qui vient combler un vide sur la question des finances des religions en Europe. L'aspect économique de la religion est en 
effet trop souvent laissé de côté alors que les implications en sont nombreuses, notamment en termes de politiques publiques ou de définition publique de la religion. Le mode de financement des Églises n'est pas non plus sans influences sur le fonctionnement même, y compris dans les activités proprement religieuses, d'une Église dans son environnement social. La lecture de cet ouvrage permet donc d'interroger différemment la situation des Églises, ou plus généralement des communautés religieuses en Europe, notamment avec la croissance de nouveaux groupes religieux qui n'étaient pas prévus dans le modèle initial de financement, par exemple l'Islam. 